\title{
THE FUTURE DIGITAL WORK FORCE: ROBOTIC PROCESS AUTOMATION (RPA)
}

Somayya Madakam https://orcid.org/0000-0001-6708-2061

FORE School of Management, New Delhi, India

Rajesh M. Holmukhe https://orcid.org/0000-0003-3696-826X

Bharati Vidyapeeth University, Pune, India

Durgesh Kumar Jaiswal https://orcid.org/0000-0003-2911-0695

National Institute of Industrial Engineering (NITIE), Mumbai, India

\begin{abstract}
The Robotic Process Automation (RPA) is a new wave of the future technologies. Robotic Process Automation is one of the most advanced technologies in the area of computers science, electronic and communications, mechanical engineering and information technology. It is a combination of both hardware and software, networking and automation for doing things very simple. In this light, the research manuscript investigated the secondary data - which is available in google, academic and research databases. The investigation went for totally 6 months, i.e., 1-1-2018 to 30-6-2018. A very few empirical articles, white papers, blogs and were found RPA and came across to compose this research manuscript. This study is exploratory in nature because of the contemporary phenomenon. The keywords used in searching of the database were Robotic Process Automation, RPA, Robots, Artificial Intelligence, Blue Prism. The study finally discovered that Robots and Robotic Process Automation technologies are becoming compulsory as a part to do business operations in the organizations across the globe. Robotic Process Automation can bring immediate value to the core business processes including employee payroll, employee status changes, new hire recruitment and on boarding, accounts receivable and payable, invoice processing, inventory management, report creation, software installations, data migration, and vendor on boarding etc. to name a few applications. Besides, the Robotic Process Automation has abundant applications including healthcare and pharmaceuticals, financial services, outsourcing, retail, telecom, energy and utilities, real estate and FMCG and many more sectors. To put in the right place of RPA in business operations, their many allied technologies are working at the background level, artificial intelligence, machine learning, deep learning, data analytics, HR analytics, virtual reality (second life), home automation, blockchain technologies, 4D printing etc. Moreover, it covers the content of different start-ups companies and existing companies - their RPA applications used across the world. This manuscript will be a good guideline for the academicians, researchers, students, and practitioners to get an overall idea.
\end{abstract}

Keywords: Automation, Robotic Automatic Process, RPA, Blue Prism, Business Process Outsourcing.

Manuscript first received: 2018-11-05. Manuscript accepted: 2018-12-03

Somayya Madakam, Assistant Professor, Information Technology Area. FORE School of Management, New Delhi, India.

E-mail: somayya@fsm.ac.in

Rajesh M. Holmukhe, Associate Professor, Department of Electrical and Engineering. Bharati Vidyapeeth University, Pune, India.

E-mail: rajeshmholmukhe@hotmail.com

Durgesh Kumar Jaiswal, Research Assistant, Centre for Technology and Innovation Management. National Institute of Industrial

Engineering (NITIE), Mumbai, India. Email: durgeshjais@nitie.ac.in 


\section{INTRODUCTION}

"Watson is creating a new partnership between people and computers that enhance, scales and accelerates human expertise"

Way back in 1943, the ENIAC, the computing machine was invented by J. Presper Eckert and John Mauchly at the University of Pennsylvania and was not completed until 1946. Some people say that abacus was the first computing machine. This is how the computing era started in decades back. Like a chameleon, the computing technology wearing different colors in terms of hardware like desktops, servers, laptops, mobiles and now entering into "rolltops". In addition to this, abundant software developments happened in terms of operating systems, applications, utilities and computing capabilities at the networking edge level with high bandwidth. Further, the organizational applications also stepped into many folds including punch cards, spreadsheets, office applications, management information systems and enterprise resource planning to assist in business applications. Nowadays the business operations are leapfrogging into the new technological land the so-called "Robotic Process Automation." Hence the year "2018" is known as the year of the "Robotic Process Automation." Industry entering into the in the new world of technology "Robotic Process Automation." known as "RPA". Timely adoption of RPA has become a necessity in day to day business activities. If Companies do not set up this technology in its operations, they may not sustain in a business competition in near future. Robotic process automation (RPA) is an emerging form of business process automation technology based on the notion of software robots or artificial intelligence (AI) workers. RPA has become the new language of business. This up to date technology is more powerful among the 21 stcentury technologies. The way Companies do their business, the way people do official work as well as the public daily life is going to be drastically assisted by new hardware, software technologies along with smart devices. The lifestyles of human being are changing across the due to global collaborations, multinational businesses, new IT/ITes advancements with RPA technology. Along with the existing new technologies functional in the human life Viz. Internet of Things, Big Data Analytics, Deep Learning, Artificial Intelligence, Machine Learning and other allied technologies, RPA is becoming one of the noteworthy disrupting technology. According to literature, to address the lack of human resources, Human-Robot Interaction (HRI) and robot companionship show positive results on the human psychological state (Banks et al., 2008; Turkle, 2006). The research on Robotic Process Automation is seriously conducted by fortune 500 companies together with new start-ups. The next section explains the meaning of Robots, Automation, and importance of Robotic Automation; followed by details of RPA.

\section{Robots}

A robot is an electromechanically designed machine, programmable by a computer and capable of carrying out a complex series of actions automatically. A robot accomplishes tasks by moving into the real world. These robots have the intelligent connections of perception to action. Artificial Intelligence must have a central role in Robotics if the connection is to be intelligent (Brady, 1985). The word "Robotics" is an interdisciplinary branch of engineering and science that includes electronics engineering, computer science, mechanical engineering, and others. For example, Sophia is a social humanoid robot developed by Hong Kong-based company Hanson Robotics. Sophia was activated on April 19, 2015 (Taylor, Harriet, 2016) and made her first public appearance by Southwest Festival (SXSW) in mid-March 2016 in Austin, Texas, United States. The report by Jump up (2018) as well reveals that Sophia is able to display more than 50 facial expressions. Gershgorn (2017) reported that according to Quartz, experts who have reviewed the robot's open-source code state that 
Sophia is best categorized as a Chabot with a face. The Sophia is the first humanoid robot to become a citizen of Saudi Arabia officially. By 2020, 10\% of large enterprises in supply-chain-dependent industries will assign one expert person a chief robotics officer role in their company operation (Douglas, and Ankush, 2017). The robotics industry has been booming in recent years. Robotics are frequently used artificial intelligence devices in the manufacturing units. The robots use wireless networking, big data, cloud computing, statistical machine learning, open-source, and other shared resources to improve performance in a wide variety of applications including assembly, inspection, driving, warehouse logistics, caregiving, package delivery, housekeeping, and surgery (Kehoe, B. et al., 2015). Swarm robotics is a novel approach to the coordination of large numbers of relatively simple robots which take its inspiration from social insects (Şahin, 2004). According to Palgrave et al. (2000), evolutionary robotics is a new technique for the automatic creation of autonomous robots. Inspired by the Darwinian principle of selective reproduction of the fittest, this technique views robots as autonomous artificial organisms that develop their own skills in close interaction with the environment without human intervention. Ambient robotics focuses on the co-adaptation and the creation of compatibility in a physical and informational sense of assistive environments and service robot systems (Linner, et al., 2014). A 3D graphics will help facility planners to visualize the system before constructing it, make alternative designs, program robot paths, obtain layouts for the systems, obtain data for the discrete event simulation, and develop the cell control program (Gunasekaran, 1999). In near future Robots are part of human society.

\section{Process}

The "Process" is the well-known term to everybody and even associated in the day to day life of people and spread across all sectors. It is the essential part of any system or firm and is the activity to accomplish a task completion. The process can be finished by people or things or a combination of both. Irrespective of the closed system or the open system, the process takes inputs from different devices or people and is completed as per the predefined rules in order to produce the desired output. The process is nothing but the conversion from input to output. However, the time taken, cost occurring, manpower required and other quality parameters vary from process to process as well as system to system. People are having familiarity with different process systems viz. biological, manufacturing, chemical, admission and many more process systems. Consider an example of a computer processor system. It takes inputs from keyboard, joystick, mouse or even with voice recognition. It processes the tasks with the help of central processing unit (CPU) or graphics processing unit (GPU) as per given commands and displays the output on the screen or printer or invoice format. The processing is happening with the help of single/ multiple core processors to carry out single/multiple tasks simultaneously. In today's advanced computing environment, companies are having cutting-edge technologies to do concurrent process very quickly and accurately within the specified constraints. All the smart devices are having the latest hardware, software and including personal digital assistants, coming up with advanced processors like Intel Core I3 - I7 8th Gen (Coffee Lake) by Intel and Ryzen 3 and 5 with RX Vega 11 Graphics by AMD are latest processors. That is why the process/processors are the essential tasks even in the exceptional technology "Robotic Process Automation".

\section{Automation}

"Automation" is the technique of making an apparatus, a process, or a system operate automatically. However, society is already reaping the automation applications in day to day life. 
Automation includes the processing capability of any system. Integrating people and systems to achieve automation is not a simple matter. Human factors, especially the cognitive aspects, are often misunderstood and neglected in system design (Sheridan, 2002). A system for generating, editing, executing, monitoring and debugging an application program for controlling an industrial automation mechanism comprising components of logic, motion and/or process control (Sadre, et al., 1996). Curb energy; is the home energy monitoring system. Ecobee3 Smart Wi-Fi Thermostats, Sonos Wireless Speaker system, Philips Hue Smart Light Bulbs, Belkin WeMo Switch and Motion Sensor, Amazon Echo device Alexa and Lutron Dimmer Light Switches are some of the recent home automated smart devices. These smart devices do the tasks by themselves with the help of embedded software and hardware. The best part of these Smart devices is that they bring the quality of life to human beings, operational efficiency and handle the situations where human being intervention is not at all possible (Madakam, 2015). The automation process makes day to day things easier, faster, and better and even free the human beings from labor and monotony of repetitive tasks. Listed are some of the most advanced automation technologies used in the automotive industry; machine vision, artificial intelligence for driverless/autonomous cars Cognitive Computing in IoT Connected Cars and collaborative robots. Automation has been already implemented in the manufacturing sector, specifically now in the form of "Industry 4.0" technology. Production assembly lines with robots doing most of the work are becoming reality.

\section{ROBOTIC PROCESS AUTOMATION}

Robotic Process Automation is the next wave of innovation, which will change outsourcing. We already are seeing the beginnings of a race to become the top automation-enabled service provider in the industry. In time, we are likely to see an arms-race for innovation in automation tools leading to new offerings and delivery models.

Sarah Burnett, Vice President of Research at the Everest Group

The term "Robotic Process Automation" connotes visions of physical robots wandering around offices performing human tasks, the term really means automation of service tasks that were previously performed by humans. For business processes, the term RPA most commonly refers to configuring software to do the work previous done by people, such as transferring data from multiple input sources like email and spreadsheets to systems of record like Enterprise Resource Planning (ERP) and Customer Relationship Management (CRM) systems (Lacity, et al., 2015). The Robotic automation is the application of specific technology and methodologies to use a computer or "virtualized FTE or robot" rather than a person to manipulate existing application software Enterprise Resource Planning's, claims applications, databases, learning management systems in the same way that a person today processes a transaction or completes a process (Sutherland, 2013). The year 2016 saw dramatic increases in the take-up of robotic process automation in back offices and shared service operations, and amongst BPO service providers themselves (Willcocks and Lacity 2016). Robotic Process Automation (RPA) moves from pilot projects to broader adoption. Interactive Voice Response (IVR) development toolkit from Pronexus itself provides basic information to the customers while connecting them to the real call center executive as per his/her requirements. M/s. Blue Prism ${ }^{\circledR}$ company, founded by David Moss and Alastair Bathgate in U.K - invented the term "Robotic Process Automation". The Blue Prism offers an intelligent, connected and easy-to-use digital workforce with new v6 features and integrations. RPA is the deployment of software to perform actions previously done by humans. The software is seen as a robot because it replaces the human resource. Combined with artificial intelligence, RPA can be used to assist in handling unstructured 
data in support of fraud / Anti Money Laundering (AML). The Shop Direct, Co-operative Banking Group, Fidelity Investments, RWE npower, the NHS and O2 to rapidly respond to business change through agile back-office operations and is powering the next major transformation in the outsourcing industry through the adoption of Blue Prisms digital labor. More CIOs are turning to an emerging technology RPA to streamline enterprise operations and reduce costs. The experts believe that with RPA, businesses can automate mundane rules-based business processes, enabling business users to devote more time to serving customers or other higher-value work (Clint Boulton, 2018). Companies are achieving productivity gains by using software robots to perform routine, rules-based service processes. If implemented well, such automation can result in high performing human-robot teams, in which software robots and human employees complement one another (Lacity \& Willcocks, 2016). For better understanding, Robotic Process Automation is the automation of repeatable and rule-based tasks by the use of non-invasive software called BOT which can mimic actions performed by human users on computers to complete various business processes (K.V.N. Rajesh et al., 2018). Although the term "Robotic Process Automation" suggests physical robots wandering around offices performing human tasks, RPA is a software-based solution. In RPA parlance, a "robot" is equivalent to one software license (Willcocks, et al., 2015). According to Gartner, RPA tools are at the "peak of inflated expectations" in the so-called Hype Cycle (Kerremans, 2018). Software tools for automated regression testing and automated performance monitoring using virtual users from different locations have been around for the past 10-15years. These tools had the ability to mimic human workers and carry out repeatable tasks like regression testing and monitoring of various front-end desktop and online applications. RPA is a natural progression of these functionalities (K.V.N. Rajesh et al., 2018). There are many vendors offering RPA tools, including Automation Edge, Automation Anywhere, Blue Prism, Cognizant, Conduent, Kofax, Kryon Systems, Pegasystems, Softomotive, and UiPath (van der Aalst et al., 2018). The physical robots have been rapidly replacing industrial, blue-collar jobs, software robots will be replacing a high percentage of white collar jobs. So it is the need of hour that the Robotic Process Automation specialist needs to know this RPA software orientation about installation, structure (Flowchart vs. Sequence), control flow (Decisions, Loops, Switches), error handling with Try/Catch, automating excel, word, and Portable Document File interactions, automating email and attachments, decomposing a process into reusable sub-components and debugging skills etc. The Robotics can generate high-quality responses to more complex questions outside the routine processes. AI techniques allow software to digest enormous amounts of research covering a huge number of possible topics-far more research than any human could. To a client asking about the outlook for the Chinese stock market, a "Chatbot" could have combined all the relevant information, reply that there was a general consensus that the Chinese stock market would rise. In fact, financial advisors, are increasingly coming under pressure from digital competitors "Robo-Advisors". According to Brandon Buccowich (2016) from M/s. Laserfiche, the Robotic Process Automation technology has many advantages including accuracy of operations, improving in employee morale, increasing the productivity, make sure low technical barriers, compliance, consistency leading to reliability and non-invasive technology. This expert had mentioned in detailed seminal about RPA advantages as given below diagram Figure 1. The beauty of robot process automation technology is that even non-technical employees will have the tools available to configure their own software robots to solve their automation challenges. He also added that RPA can bring immediate value to the core business processes including payroll, employee status changes, new hire recruitment and on boarding, accounts receivable and accounts payable, invoice processing, inventory management, report creation, software installations, data migration, and vendor on boarding etc. to name a few. 


\section{Robotic Process Automation Operations}

There are no standard operational models developed still now for the Robotic Process Automation operations. However, Mr. Kristina Romero and his technical team from M/s. InfoCap Networks LLC (InfoCap), San Diego, propose a sample of Robotic Process Automation operations as given in Figure 2. This technological model will replace the entire business system operations by automating manual work, more into human intensive, time consuming, specifically error prone content enabled business (Kristina Romero, 2017). In this sense, the main advantage is the "Digital Labour" to reduce costs, minimize errors, and eliminate the risks. The RPA has many operational advantages across multidisciplinary and varied organizations.

Credible Business Transformation: With the new RPA technology, the business operations are going to be changed drastically. The companies can now dramatically improve the efficiency with which they use their labor, augmenting a more sustainable workforce with reliable, efficient and low-cost digital labor using Robotic Process Automation. This allows companies to reduce costs, minimize errors, and eliminate risk.

Content Migrations: A huge amount of content is generating in all the organizations. Manpower may be required to collect, analyze, and generate a report as insights is becoming complex in routine operations. Robotic Process Automation can only help the organizations and business companies to accelerate application consolidation and legacy application integration by migrating content or connecting to legacy systems more rapidly, with less effort.

Web Crawling / OSINT: Robotic Process Automation automates the capture of content in any format from any source through various devices. The formats may be in text, picture, audio, video. The

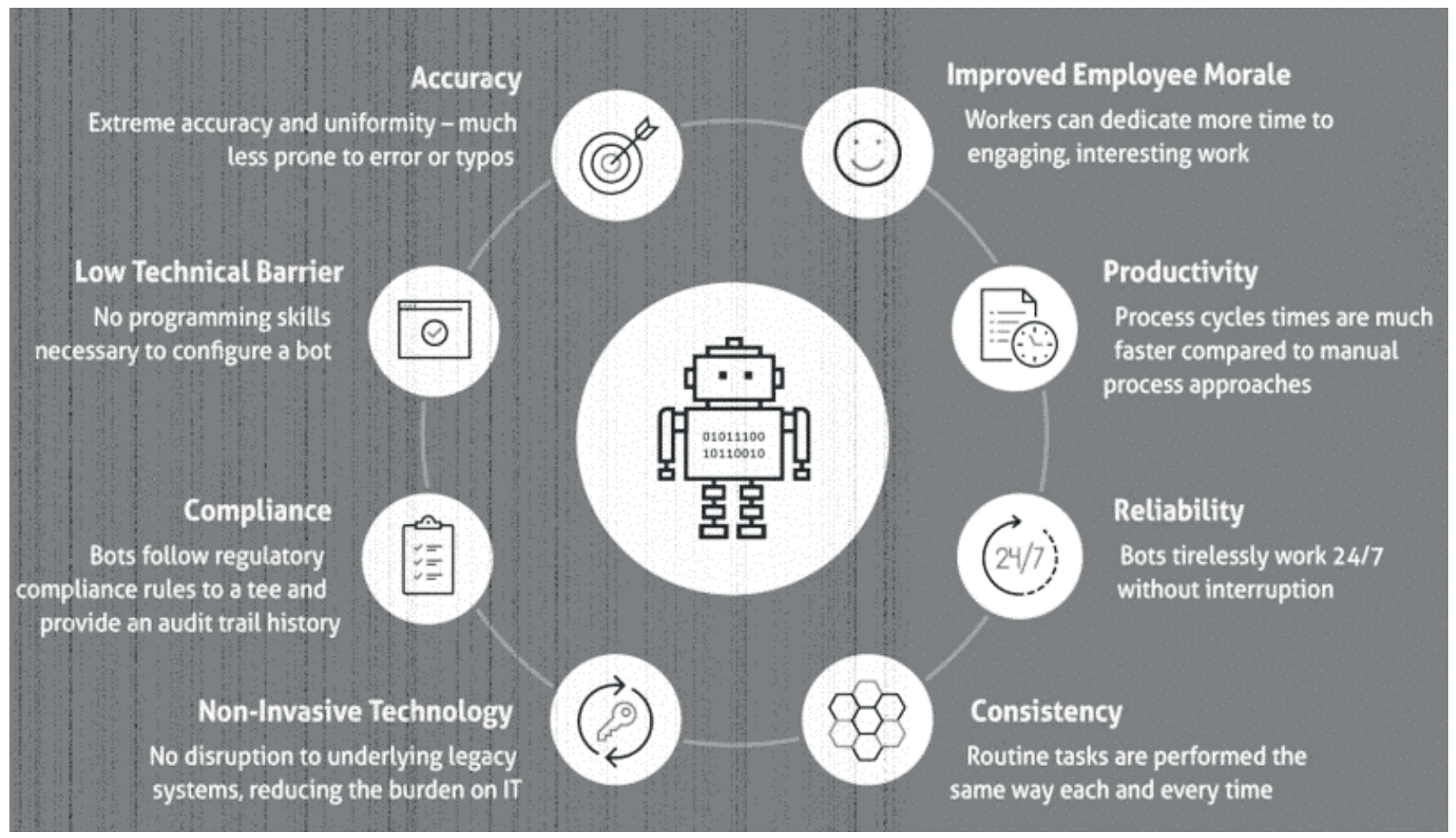

Figure 1. Robotic Process Automation, by Brandon Buccowich. 


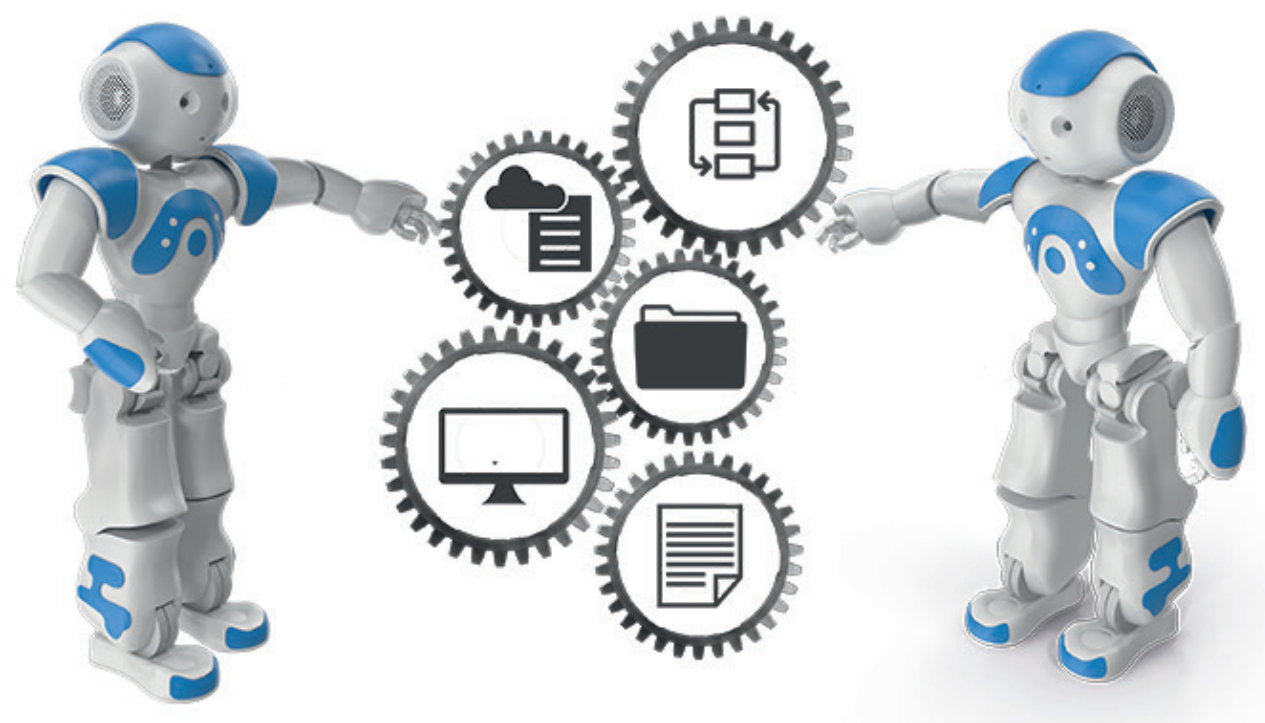

Figure 2. RPA Operations, by Kristina Romero (2017).

data can be the structured, semi-structured and unstructured format. This Robotic Process Automation technology is capable of harvesting deep web data via deep learning techniques. Moreover, with the help of Artificial Intelligence, Big Data Analytics, and other web analytics, the mining process will have done.

IT Department Enabler: In their blog, they mentioned that the "Robots" in the Robotic Process Automation "Software programs that mimic human-computer interactions and execute a repetitive process, rules-based tasks like gathering and comparing data from multiple systems, reading and writing to databases, or extracting and reformatting data into reports and dashboards". They keep on the eye in hardware and software and networking monitoring for resolving deviations and smooth operations.

\section{RPA-Operating Model Design}

Robotic Process Automation is an emerging form of business process automation technology based on the notion of software robots or artificial intelligence workers. Rodger Howell and Tom Torlone (2017) in their seminal article insisting the growth of Robotic Process Automation technology. They rightly pointed out that the Robotic Process Automation is coming in reality from pilot projects. Hence the organizations need to define their own RPA models to increase the operational efficiency and reduce the cost. These authors mean to say that the RPA operational models vary from companies to companies and industry to industry. In their opinion, the Robotic Process Automation operating models are not "one-size-fits-all". However, an effective RPA operating model is largely cantered around three key roles.

\section{Process Architects}

Who help design future state processes empowered by Robotic Process Automation. The process is nothing but task completion. It is associated with many factors of software like Interactive Voice Response, hardware like robots, few human interventions. There are different types of the process including of long-term and short-term process/round robin, priority / First Come First Served 
Process (FCFS) / Last In First Out (LIFO). The process architects are responsible for defining all these processes in both the centralized process and decentralized process systems. First, they have to understand the existing system flow, identify the loopholes in the system components, tasks handling, time requirements, and cost reductions along with systems efficiency. In one way is the business analysts are also responsible for process automation. The process architects design the consistent methodology, process, and standards of Robotic Process Automation system.

\section{Technologists}

Who develop the code that translates the business logic into a robotic workflow? We know that a technologist is a scientist or an engineer who specializes in a particular technology, or who uses technology in a particular field. In the market right now the companies are having more. These are the coders, programmers, they take the input from functionalist and designers, executors and develop the code based on the Software Requirement Specifications (SRS). All these coded software is able to help the routine work handling automatically without human interventions at a certain level. The authors also point out that a Robotic Process Automation Centre of Excellence will usually put a team of dedicated developers in a low-cost location to scale a Robotic Process Automation program. The technical skills required for RPA tools are relatively less sophisticated compared to traditional application development.

\section{Ongoing Support and Maintenance Staff}

Who execute newly automated tasks and make updates to the code when required. This process happens generally through annual maintenance contract (AMC) with the software vendor or supplier. They easily debug the errors if any fault and failure in the system software and applications. They give technical support $24 \times 7$ round the year if AMC is valid. This kind of technical support really reduces the in-house technical people recruitments cost and time. There would not be any training cost also. The ongoing support packages from the companies are tailored to suit the needs of customer business, not the needs of the many. Whether customer need assistance with an individual problem or weekly reviews and advice have the package to suit customer's needs, giving customer total peace of mind.

\section{RPA Application in Airtel}

One of the best applications of Robotic Process Automation is Business Process Outsourcing. The same kind of routine work with technical support is provided by software by replacing the many technical staff $24 \times 7$. The clients may be located in different parts of the country. This is the best application that reduces the manpower for the repeated and continuous kind of operations. However, some manpower is kept for the inevitable tasks. For example, in the Airtel company in India, the technical support is provided by the company primarily by use of software for the basic and routine tasks. The same is easy of doing the tasks in the local languages also. Any customer can dial the tollfree number for any kind of the help from Airtel operators. The above Figure 3 show in-detail Robotic Process Automation Process. There are many sorts of applications and problems in terms of mobile usage. Hence, the software helps in terms of language, payments, caller-tunes, ringtones, internet data usage, sim-lost, new tariff plan etc. All these options are very simple and are executed just by typing one of the 1-8 options, in the number pad depending on the features in option and through voice input support will be provided. 


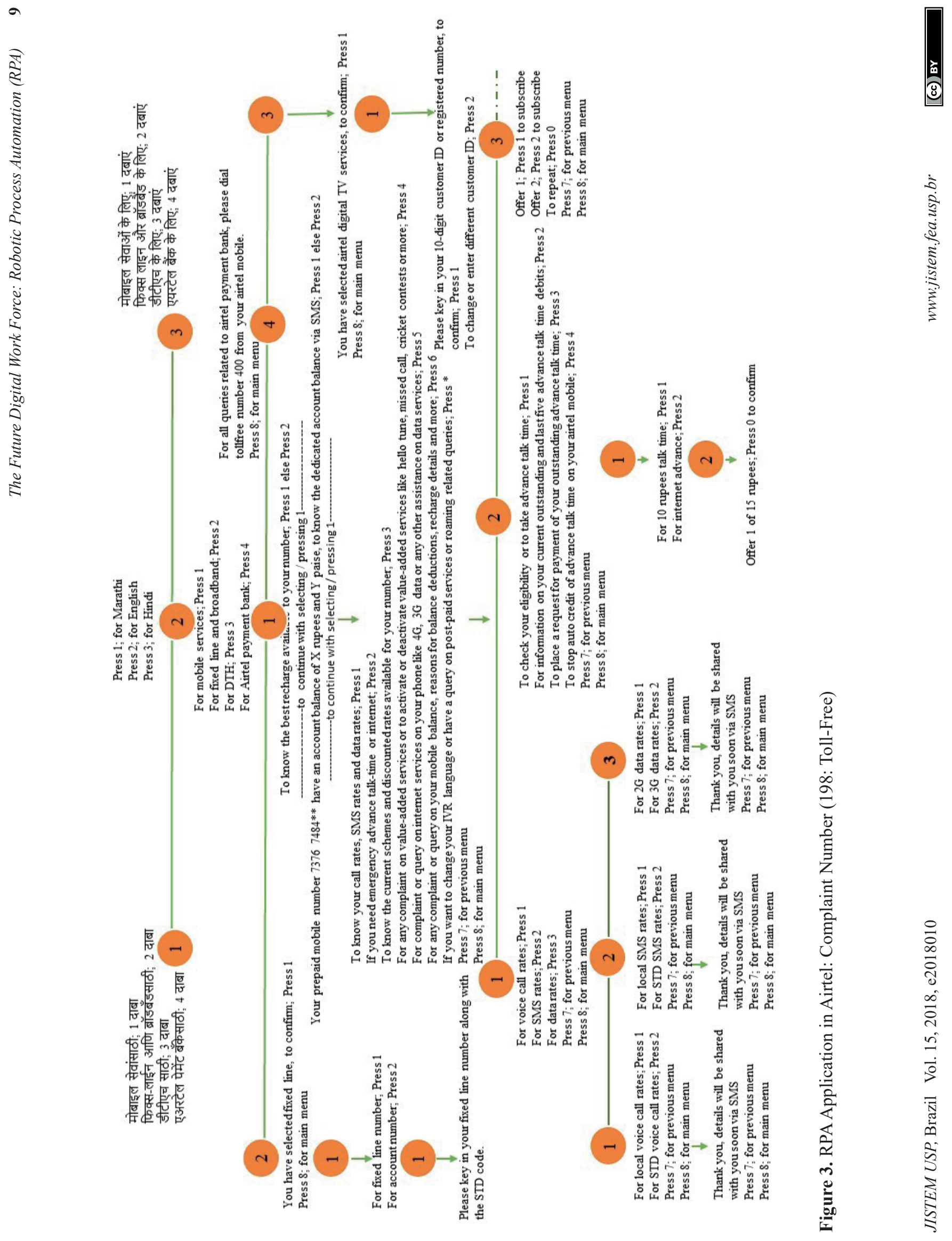




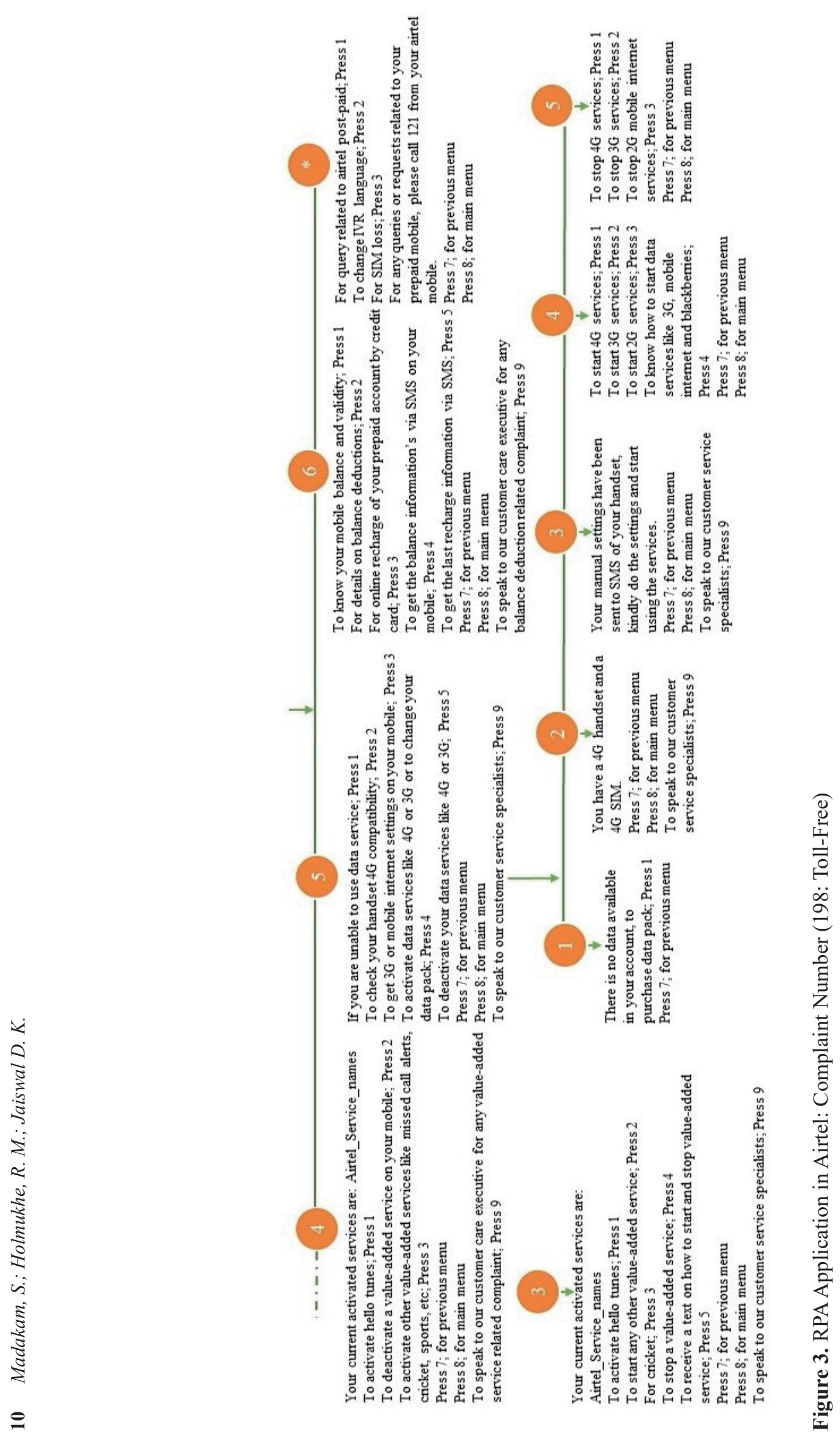

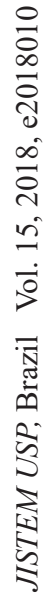




\section{WHO IS WHO IN RPA BUSINESS}

The advancement in hardware, software especially including Artificial Intelligence (AI) paved way for Robotic Process Automation, which has the capability to reimagine the new business management landscape. As rightly pointed out by Willcocks and Lacity (2016), Robotic Process Automation and cognitive automation has the potential to be very disruptive of the more conventional people-centric outsourcing model that offshore outsourcing vendors and captive centres were based on. Undoubtedly in each round of contract renewals across 2017-2020, we will see clients make new demands - on cloud computing, robotic process automation, as-a-service, and adoption of new technologies and business analytics, and industry will see providers adjusting their services to reflect these developments (Willcocks, et al., 2017). Over the years, RPA has been adopted by a number of Business Process Outsourcing (BPO) providers and a growing number of end-user organizations are now deploying the technology themselves to create "virtual workforces" of robotic workers. Robotic Process Automation describes the use of technology to automate tasks that are traditionally done by a human being. The technology itself mimics an end user by simulating user actions such as navigating within an application or entering data into forms according to a set of rules (Barnett, 2015). RPA is part of the spectrum of emerging Artificial Intelligence tools, including virtual agents, machine learning, computer vision and natural language classification. The move to artificial intelligence technologies can have many applications in insurance, for instance, the image classification for claims and text analytics for servicing customer queries. These new technologies will further drive automation and augmentation of insurance processes.

Blue Prism Version 6 is a digital workforce operating system software, developed by M/s. Blue Prism. This company is pioneered in Robotic Process Automation software. Their thought process is that effective digital strategies require a connected enterprise, where technologies such as artificial intelligence (AI), machine learning (ML) and sentiment analysis easily integrate with business operations to deliver value. The Blue Prism v 6 software platform enables the business operations to be agile and cost-effective through rapid automation of manual, rules-based, back office administrative processes, reducing cost and improving accuracy by creating an efficient "digital workforce". Some of the best solutions which are addressed by the Blue Prism's Robotic Process Automation software are

- Rules-based processing with digital structured inputs such as credit card activation or fraud checking

- Repetitious transactional processing such as SIM swaps or invoice processing

- Complex/mission critical processing - pension redemptions and financial reconciliation

- High transaction volumes such as billing or new handset order processing

- Process adherence/quality issues such as policy renewals or policy migrations

- Fluctuation in demand or backlogs such as with new product launches

- "Swivel Chair" processes such as Human Resource on boarding or launching a new online product where there is no integration

$M / s$. UiPath is in the front position of the digital business revolution across the globe by enabling the rapid deployment of software robots that dramatically improve business productivity, 
compliance and customer service across back-office and front-office operations. UiPath believes that Robotic Process Automation systems log into applications, move files and folders, copy and paste data, fill in forms, extract structured and semi-structured data from documents, scrape browsers, what not many more. Accel, CapitalG, and Kleiner Perkins Caufield \& Byers invested in UiPath worth of a \$153 million investment, valuing the company at \$1.1 billion as dated March 6, 2018. Another best example in the business of RPA, M/s. Automation Anywhere is the cognitive robotic process automation platform designed to automate any business process in the modern enterprise. The Automation Anywhere Bot Store from Automation Anywhere company enables organizations to scale up their digital workforce faster than ever before. This enhances the productivity of human workers and allows them to focus on more human-centric initiatives. M/s. Automation Anywhere company has to provide the world's best Robotic Process Automation and cognitive technology to leading healthcare, financial services, technology, manufacturing and insurance firms for over a decade.

$M / s$. Endpoint Automated Services (EAS) is another UK based specialist automation and cloud consultancy company is bringing its extensive experience in the world of automation to the Robotic Process Automation discipline. For instances of robot, automation is the execution of possibly automating a full end-to-end business process and data entry into a financial accounting system. Robotic Process Automation has been used to automate even the most complex of processes even the Microsoft Office suite of applications. RPA is a notable codeless automation. The robots can be taught on a screen scraping basis and again the robots are taught how to find anchors on the screen as opposed to using restrictive pixel based coded screen scrape. The M/s. Endpoint Automated Services company believe that

"Robotic Process Automation delivers direct profitability while improving accuracy across organizations and industries. Designed to perform on a vast range of repetitive tasks, software robots interpret, trigger responses and communicate with other systems just as humans do. Only substantially better: a robot never sleeps, makes zero mistakes and costs a lot less than an employee"

David Silver, Dean Hough (1985) founded M/s. Kofax Inc. (Kofax) company. It is a process automation software provider located at international headquarters in Irvine, California. Kofax provides process automatically extract and classify data from paper and digital sources, extracted data is transferred to appropriate business departments and the result is streamlined data processing with reduced human intervention and error. As the demand for single platform software with mobile applications increased, Kofax introduced Document Workflow, Imaging, Intelligent Capture, and Business Process Management.

$M / s$. "O2" is the United Kingdom (UK) based telecommunications company. It is one of the leading digital communications company in the UK with over 25 million customers, has over 450 retail stores and sponsors. The $\mathrm{O} 2$ company runs its business with $2 \mathrm{G}, 3 \mathrm{G}$ and $4 \mathrm{G}$ networks across the United Kingdom along with operating O2 Wireless Fieldality (Wi-Fi) and owning half of Tesco Mobile. As per 31st, March 2015, during the period from 2012-2015, means those three years, O2 has replaced 150 workers with a single piece of automated software. Mr. Wayne Butterfield, who works on $\mathrm{O} 2$ operations reported that a great portion of O2's customer service is now automatic. He also added, "Sim swaps, porting mobile numbers, migrating from prepaid onto a contract, unlocking a phone from O2." This is a good real-time application of RPA. The Cap Gemini in-house robots have processed 1,5 Million transactions since 2015, equivalent to 200 employees, "Process design is 
more relevant to the Return on Investment than the technology used". David Schatsky, a managing director@ Deloitte LP, pointed that to a bank's experience with implementing RPA technology, in which the bank redesigned its claims process by deploying 85 bots to run 13 processes, handling 1.5 million requests per year. The bank added capacity equivalent to more than 200 full-time employees at approximately 30 percent of the cost of recruiting more staff (Clint, 2018). The author also reported that AT\&T, Walmart, Deutsche Bank, Ernst \& Young, Vanguard, Walgreens, Anthem and American Express Global Business Travel are among the many enterprises adopting RPA technology.

The Robotic Process Automation has abundant applications in all over the domains including healthcare and pharmaceuticals, financial services, outsourcing, retail, telecom, energy and utilities, real estate and FMCG (Fast Moving Consumer Goods) and many more. Besides, some of the Robotic Process Automation technologies can perform include the corporate activities across finance, compliance, treasury, and marketing including of data cleaning, order management, claims handling, payment processing, direct debit, compliance report writing, underwriting in banking and automated marketing campaigns. That is why the new employment profiles by Robotic Process Automatic systems are changing into transcribers, financial analysts, taxi drivers, home automation, senior citizen assistance and patient care etc. If we go for one of the pioneers in Robotic Products solutions, they provide varied solutions including on premise or cloud-based solution, managed service capability, certified specialists to help in automation, process consultancy expertise, non-disruptive solutions, no replacement of systems required, no software development or system integration, improves speed and consistency, significantly reduces costs, and frees staff to do the things that really need them. Some of the RPA solutions which are developed and tested by M/s. Infosys, Indian based company includes are by blending automation and artificial intelligence, Infosys BPM reimagines business process management and delivers an innovative business process service stack. One that has been built with thousands of hours of learning and testing, and shaped to perfection in varied and complex client environments. These are some of the multinational companies' products and solutions relating to Robotic Process Automation technologies. In addition, many others companies also set out to design, develop, implementing RPA technologies including new start-up companies. However, to put in the right place of RPA in business operations, there other allied technologies are working at the background level, artificial intelligence, machine learning, deep learning, data analytics, HR analytics, virtual reality (second life), home automation, blockchain technologies, 4D printing etc.

M/s. Everest Group - RPA Future: M/s. Everest Group company reveals that the Robotic Process Automation has many applications across the many industries including banking \& financial services of cards activation, frauds claims discovery, insurance services of claims processing and new business preparation, healthcare sector possessing with reports automation and system reconciliation, manufacturing firms with specifically bills of material (BoM) generation, high tech \& telecom fields with service order management quality reporting and Energy Utilities account setup plus meter reading validation especially as shown in the Figure 4. Moreover, the report also discovers that RPA applications have high usage in banking and manufacturing sectors then followed by insurance, healthcare, High Tech \& Telecom and energy utilities in terms of accounts payable, accounts receivables, and general ledger processing. Whereas cards activation, frauds claims discovery, claims processing, new business preparation, reports automation and system reconciliation process to have the high potential in banking and financial services, insurance and healthcare sectors. This we can find in the below figure. Everest Group company predicts the Robotic Process Automation (RPA) applications market has huge potential in the future across the globe. 


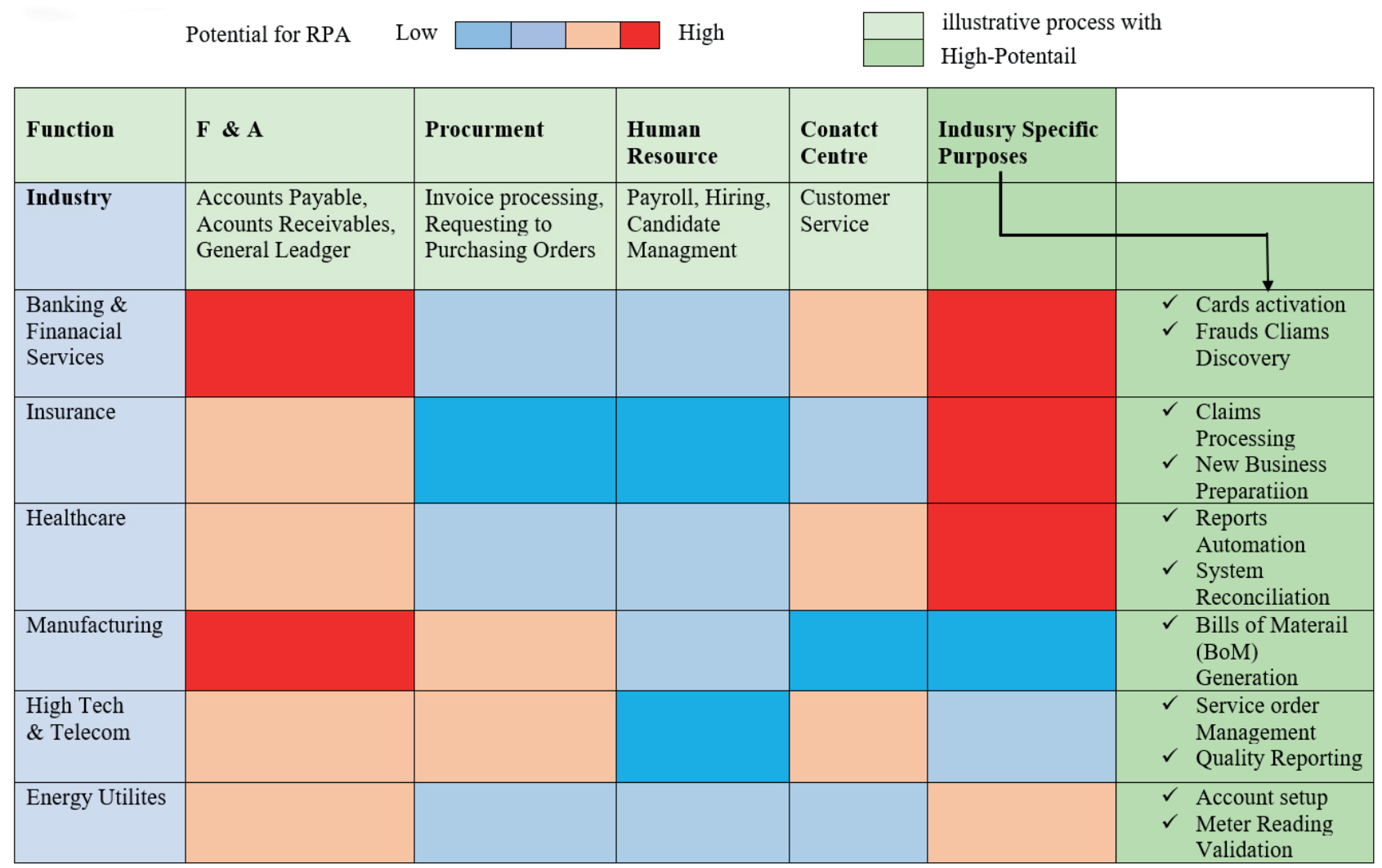

Figure 4. RPA Adoption by Buyer Industry and Function.

The global market for RPA technology products and services would pass around $\$ 400$ million by the year 2017. It is expected to grow at a compound annual growth rate of $36 \%$ to almost $\$ 1.2$ billion by 2021. The direct services market includes implementation and consulting services focused on building RPA capabilities within an organization. It also includes Bots, algorithms, and the future of the finance functions in all organizations and banks. Automation and artificial intelligence are poised to reshape the finance function. It means a lot of automated devices will be mounted in the financial institutions, banks to take or carry out routine operations, which will almost reduce operational cost, $24 \times 7$ online services, and security. These bots moreover bring accuracy in operations and are very user-friendly even for illiterate people with their mother tongue. In India State Bank of India have already started mounting bot technology for financial services. According to Gartner's prediction by the year 2020, consumers will manage their $85 \%$ of the relationships with the enterprises without interacting with a human. That means there will be a huge amount of automation in each industry.

\section{CONCLUSION}

Robots and Robotic Process Automation technologies are becoming compulsory as a part to do business operations in the organization. They will do recruitment of the employees, screening as well as selecting people, give induction and training to the newly recruited and even look for the employee welfare schemes of the organization along with doing traditional repetitive tasks. In some firms, the robots will be replacing completely the manpower. The best part is that they will do repetitive tasks and even involve in the risky process of human life. So the human resources in the organizations can 
give their valuable time in prime decision making or the intelligent process of the company. Like the Sophia, the humanoid and her upcoming sister humanoids will assist even in human's personal daily life. The greatest part of this Robotic Process Automation technology is that it is more convenient and more helpful to the elderly lonely people plus differently abled candidates round the clock. Across the globe many sectors already trying to deploy these Robotic Process Automation technologies including manufacturing industries, chemical plants, the healthcare sector, aviation and so on, that were already discussed in this article. Let industry welcome the artificial intelligence humanoids to assist to human life. With this technology people will be improving their quality of life, caring of elderly people, get services in $24 \times 7$, escape the risky jobs from dangerous situations, bust workflow inefficiencies, shoring up productivity, and free people from doing repetitive tasks.

\section{METHODOLOGY}

Robotic Process Automation is one of the most advanced technologies in the area of Computers Science and Information Technology. Robotic Process Automation is a just begotten phenomenon. Nobody knows exactly what does it mean, there are no standard operational definitions, connotes, tested models, and derived theories. It means it is exploratory in nature. As a result, the data used to compose this research article is taken from different secondary sources (on-line), i.e. research manuscripts, corporate white papers, expert's blogs, thematic videos etc. The data was gathered from the period of April 2018 to June 2018. The keywords used to fetch the research articles are "Robots", "Robotic Process Automation", "Artificial Intelligence", and "AI". The search engines Google and Google scholar are used for articles in the databases. The ancillary data was collected, collated, analyzed and thematic narration done for better understanding the Robotic Process Automation phenomenon. This study comes under exploratory study, inline to descriptive research.

\section{REFERENCES}

Banks, M. R., Willoughby, L. M., \& Banks, W. A. (2008). Animal-assisted therapy and loneliness in nursing homes: use of robotic versus living dogs. Journal of the American Medical Directors Association, 9 (3), $173-177$.

Barnett, G. (2015). Robotic process automation: adding to the process transformation toolkit.

Blue Prism Software Robots: Introducing the Virtual Workforce. Accessed dated on 12/6/2018 from the https://www.blueprism.com/about-us.

Brady, M. (1985). Artificial intelligence and robotics. Artificial intelligence, 26(1), 79-121.

Brandon Buccowich (2016). What is Robotic Process Automation. Accessed dated on 20/6/2018, https:// www.laserfiche.com/ecmblog/what-is-robotic-process-automation-rpa/.

Clint Boulton (2018). What is RPA? A revolution in business process automation, https://www.cio.com/ article/3236451/business-process-management/what-is-rpa-robotic-process-automation-explained.html.

Douglas Laney, Ankush Jain (20 June 2017). 100 Data and Analytics Predictions Through 2021.Accessed dated on 13/6/2018 from the Universal Resource Locator file://C:/Users/somayya/AppData/Local/ Packages/Microsoft.MicrosoftEdge_8wekyb3d8bbwe/TempState/Downloads/100\%20Data\%20and\%20 Analytics\%20Predictions\%20Through\%202021.pdf.

Gershgorn, Dave (12 November 2017). "Inside the mechanical brain of the world's first robot citizen”. QZ. Retrieved 13 November 2017. 
Gunasekaran, A. (1999). Agile manufacturing: a framework for research and development. International journal of production economics, 62(1), 87-105.

Hal Hodson (2015). AI interns: Software already taking jobs from humans. Accessed dated on 12/6/2018 from the https://www.newscientist.com/article/mg22630151.700-ai-interns-software-already-taking-jobsfrom-humans/\#.VY2CxPlViko

Harness the Power of Automation: Discover how Endpoint Automation can automate your IT processes and save you money, accessed dated on 12/6/2018 from the URL http:/www.endpointautomation.co.uk/.

Jump up "Meet Sophia, the female humanoid robot and newest SXSW celebrity". PCWorld. Retrieved 201801-04.

K.V.N. Rajesh, K.V.N. Ramesh and Hanumantha Rao (2018). Robotic Process Automation: A Death knell to dead-end jobs? CSI Communications-Knowledge Digest for IT Community, Volume No.42, Issue No.3, $10-14$

Kehoe, B., Patil, S., Abbeel, P., \& Goldberg, K. (2015). A survey of research on cloud robotics and automation. IEEE Transactions on automation science and engineering, 12(2), 398-409.

Kerremans M (2018) Gartner market guide for process mining. Report G00353970. Gartner.

Kristina Romero (December 15, 2017). Content Enabled Robotic Process Automation and Analytics. Accssed from https://infocapnet.com/robotic-process-automation/ on 23/7/2018.

Lacity, M., Willcocks, L. P., \& Craig, A. (2015). Robotic process automation at Telefonica O2.

Lacity, M. C., \& Willcocks, L. P. (2016). A new approach to automating services. MIT Sloan Management Review, 58(1), 41.

Linner, T., Pan, W., Georgoulas, C., Georgescu, B., Güttler, J., \& Bock, T. (2014). Co-adaptation of robot systems, processes and in-house environments for professional care assistance in an ageing society. Procedia Engineering, 85, 328-338.

Madakam, S. (2015). Internet of things: smart things. International Journal of Future Computer and Communication, 4(4), 250.

M.C. Lacity and L.P. Willcocks, “Advanced Outsourcing Practice: Rethinking ITO, BPO, and Cloud Services" (London: Palgrave, 2012).

Palgrave Macmillan, Cham. Nolfi, S., \& Floreano, D. (2000). Evolutionary robotics: The biology, intelligence, and technology of self-organizing machines. MIT press.

Sadre, A., Baechtel, D. F., \& Graber, M. S. (1996). U.S. Patent No. 5,485,620. Washington, DC: U.S. Patent and Trademark Office.

Rodger Howell and Tom Torlone (2017). Robotic Process Automation (RPA) Operating Model Design. Accessed dated on 13/7/2018 from https://www.linkedin.com/pulse/robotic-process-automation-rpaoperating-model-design-rodger-howell/.

Şahin, E. (2004, July). Swarm robotics: From sources of inspiration to domains of application. In International workshop on swarm robotics (pp. 10-20). Springer, Berlin, Heidelberg.

Sheridan, T. B. (2002). Humans and automation: System design and research issues. Human Factors and Ergonomics Society.

Sutherland, C. (2013). Framing a Constitution for Robotistan. Hfs Research, ottobre.

Taylor, Harriet (2016-03-16). “Could you fall in love with robot Sophia?”. CNBC. Retrieved 2018-01-04. 
Turkle, S. (2006). A nascent robotics culture: new complicities for companionship. In American Association for Artificial Intelligence Technical Report Series AAAI.

van der Aalst, W. M., Bichler, M., \& Heinzl, A. (2018). Robotic Process Automation.

Willcocks, L. P., Lacity, M., \& Craig, A. (2015). The IT function and robotic process automation.

Willcocks, L., \& Lacity, M. (2016) Service Automation, Robots and The Future of Work. SB Publishing, Stratford.

Willcocks, L. P., Lacity, M. C., \& Sauer, C. (2017). Introduction. In Outsourcing and Offshoring Business Services (pp. 1-22). 